\title{
The unusual Nova Cygni 2006 (V2362 Cygni)
}

\author{
S. Kimeswenger, S. Dalnodar, A. Knapp, J. Schafer, S. Unterguggenberger, and S. Weiss
}

\author{
Institut für Astro- und Teilchenphysik, Universität Innsbruck, Technikerstraße 25, 6020 Innsbruck, Austria \\ e-mail: stefan.kimeswenger@uibk.ac.at
}

Received 9 October 2007 / Accepted 11 January 2008

ABSTRACT

\begin{abstract}
Context. Optical nova lightcurves often have structures, such as rapid declines and recoveries, due to nebular or dusty phases of the ejecta. Nova Cygni 2006 (V2362 Cyg) underwent an unusual brightening after an early rapid decline. The shape of the lightcurve can be compared to that of V1493 Aql, but in that case the whole event was not as bright and only lasted a couple of weeks. V2362 Cyg had a moderately fast decline of $t_{2}=9.0 \pm 0.5$ days before rebrightening, which lasted 250 days after maximum.

Aims. We present an analysis of our own spectroscopic investigations in combination with American Association of Variable Star Observers (AAVSO) photometric data covering the whole rebrightening phase until the return to the final decline.

Methods. We used medium resolution spectroscopy obtained in ten observing nights in the course of 79 nights to investigate the change of the velocity structure of the ejecta. The publicly available AAVSO photometry was used to analyze the overall properties and the energy of the brightening.

Results. Although the behavior of the main outburst (velocity, outburst magnitude, and decline timescales) resembles a "normal" classical nova, the shell clearly underwent a second fast mass ejecting phase, causing the unusual properties. The integrated flux during this event contributes $\approx 40 \%$ to the total radiation energy of the outburst. The evolution of the $\mathrm{H}_{\alpha}$ profile during the bump event is obtained by subtracting the emission of the detached shells of the main eruption by a simple optically-thin model. A distance of $D \approx 7.5_{-2.5}^{+3.0} \mathrm{kpc}$ and an interstellar extinction $E(B-V)=0.6 \pm 0.1$ was also derived.
\end{abstract}

Key words. stars: novae, cataclysmic variables - stars: individual: Nova Cygni 2006 = V2362 Cyg

\section{Introduction}

Nova Cygni 2006 (V2362 Cyg) was discovered April 2, 2006 by H. Nishimura as a $10 \mathrm{~m} .5$ object (Nakano et al. 2006). The early spectra, obtained $\approx 0.6$ prior to the maximum in $V$, displayed a rather flat continuum, over which the $\mathrm{H}_{\alpha}$ line shows a clear P-Cyg profile. The FWHM of the emission was $500 \mathrm{~km} \mathrm{~s}^{-1}$ and the absorption minimum was $700 \mathrm{~km} \mathrm{~s}^{-1}$ blueshifted (Yamaoka et al. 2006). Within a day ( $\approx-0.2)$, the profiles showed two absorption features at -880 and $-1730 \mathrm{~km} \mathrm{~s}^{-1}$ (Czart et al. 2006). Based on spectra obtained on April 13, $2006(\approx 6.7)$, Siviero et al. (2006) classified the nova as "Fe-II" and found a very broad and structured profile with FWZI of $3750 \mathrm{~km} \mathrm{~s}^{-1}$ and $F W H M$ of $1800 \mathrm{~km} \mathrm{~s}^{-1}$ from the Balmer emission lines.

With the exact position found by Yamaoka (2006), Steeghs et al. (2006) identified the progenitor as an emission line star in the IPHAS $\mathrm{H}_{\alpha}$ survey. With progenitor magnitudes of $r^{\prime}=$ $20 . \mathrm{m} 3 \pm 0.05$ and $i^{\prime}=19 \mathrm{~m} .76 \pm 0.07$ the outburst amplitude was about 12 mag.

In July $2006\left(\approx 100^{\mathrm{d}}\right)$ the first unusual behavior was reported by Mazuk et al. (2006). The nova, after having already declined by $4^{\mathrm{m}}$, still showed only low excitation lines. The target brightened (Munari et al. 2006a) from August $2006\left(\approx 130^{\mathrm{d}}\right)$ until beginning of December $2006\left(\approx 240^{\mathrm{d}}\right)$. Goranskij et al. (2006) found an optical period of 0.2070 and an increase of the emission line width during this phase. They also pointed out similarities to the lightcurve of V1493 Aql (Nova Aql 1999a). The second maximum in V1493 Aql had a shorter duration of 2 weeks and a smaller amplitude of only $\approx 0$. 75 . The duration in V2362 Cyg was 6 months with an amplitude of 2 . 1 . At the beginning of December 2006 the brightness dropped by 2.5 over a period of two weeks to a value expected from the extrapolation of the early decay. The spectra also changed rapidly (Kimeswenger et al. 2006) and reached a typical nebular phase at the end of December 2006 (Munari et al. 2006c). The formation of very hot dust, $T_{\mathrm{d}} \approx 1410 \mathrm{~K}$, began before December $12,2006\left(\approx 250^{\mathrm{d}}\right)$ (Lynch et al. 2006; Rayner et al. 2006). In May $2007\left(\approx 396^{\mathrm{d}}\right)$, Lynch et al. (2007) reported continuing dust emission but at a lower temperature, $T_{\mathrm{d}}<520 \mathrm{~K}$.

Ness et al. (2006) also detected the source in X-rays on October 14, $2006\left(\approx 191^{\mathrm{d}}\right)$, and reported that the spectrum is harder than expected from supersoft X-ray binaries. On May 5, $2007\left(\approx 394^{\mathrm{d}}\right)$, Hernanz et al. (2007) reported that the XMM-Newton spectra were poorly fitted with a single component absorbed model. They fitted a two-temperature thermal plasma model with a low and a high temperature of $0.2 \mathrm{keV}$ and $2.3 \mathrm{keV}$, respectively.

Here we report the results of a spectral monitoring throughout the main brightening phase and further on until the target reached its final decline.

\section{Data}

We obtained the spectroscopic data in ten nights in the period from early October 2006 until the end of December 2006 at the $60 \mathrm{~cm}$ telescope of the University of Innsbruck. We used the 10C slit spectrograph with a $50 \mu \mathrm{m}$ slit and the $240 \mathrm{l} / \mathrm{mm}$ grating. The sampling at the Kodak $0400 \mathrm{CCD}$ was $0.26 \mathrm{~nm} / \mathrm{pixel}$ and the resolution, measured as $F W H M$ of the night sky lines, was $0.6 \mathrm{~nm}$. The combined frames typically covered 400-900 $\mathrm{nm}$ each night. The observational log is given in 
Table 1. Observing log of spectroscopy and wavelength range covered.

\begin{tabular}{lllc}
\hline \hline Date & $\begin{array}{l}\text { Wavelength } \\
\text { range [nm] }\end{array}$ & Observers & Day $^{*}$ \\
\hline 10th Oct. & $400.9-573.5$ & $\mathrm{KS}$ & 187 \\
& $591.4-791.6$ & & \\
16th Oct. & $400.0-900.0$ & $\mathrm{SD}, \mathrm{JS}, \mathrm{SU}, \mathrm{KS}$ & 193 \\
17th Oct. & $400.0-900.0$ & $\mathrm{KS}$ & 194 \\
15th Nov. & $400.0-893.5$ & $\mathrm{SD}, \mathrm{AK}, \mathrm{JS}, \mathrm{SU}, \mathrm{SW}, \mathrm{KS}$ & 223 \\
20th Nov. & $400.0-736.7$ & $\mathrm{KS}$ & 228 \\
7th Dec. & $400.0-900.0$ & $\mathrm{KS}$ & 245 \\
13th Dec. & $400.0-900.0$ & $\mathrm{SD}, \mathrm{AK}, \mathrm{JS}, \mathrm{SU}, \mathrm{SW}, \mathrm{KS}$ & 251 \\
14th Dec. & $400.0-900.0$ & $\mathrm{SD}, \mathrm{SU}$ & 252 \\
23rd Dec. & $537.5-740.0$ & $\mathrm{KS}$ & 261 \\
27th Dec. & $400.0-740.0$ & $\mathrm{KS}$ & 265 \\
\hline
\end{tabular}

* Days since maximum in AAVSO data April 06, 2006 09:33 UT (JD 2453 831.8983), SD = Silvia Dalnodar, AK = Andreas Knapp, JS = Josef Schafer, $\mathrm{SU}=$ Stefanie Unterguggenberger, $\mathrm{SW}=$ Sarah Weiss, $\mathrm{KS}=$ Stefan Kimeswenger.

Table 1. For a more detailed description of the instrument see Kimeswenger et al. (2003) and references therein.

We obtained the flux calibration using Vega (HR 7001), HR 8252, and HR 8079. The calibration was verified by folding the resulting spectra with standard $V$ and $I_{C}$ filter curves. The zero points were $24^{\mathrm{m}} .23$ and $23^{\mathrm{m}} .12$ in $V$ and $I_{\mathrm{C}}$, respectively. The rms of the photometric nights was 0.056 . The nights of November 20, December 7, and 27, 2006 were not photometric. We corrected the spectra to fit to the published photometries for those three nonphotometric nights. The resulting zero points are consistent with the observational average.

The photometric data was obtained from the AAVSO data base (Henden 2007) and various circulars (Munari et al. 2006a-c; Goranskij et al. 2006). We only used CCD-based photometry with filters for the lightcurves shown in Fig. 1. The first values with CCDs and a $V$ band filter were reported April 5, 2006 6:56 UT (JD 2453 830.7891). The maximum value of 7. 8 was reached April 6, 9:33 UT (JD 2 453 831.8983). We used this value as a "reference zero point" for all calculations. The decline derived from there was $t_{2}=9^{\mathrm{d}} .0 \pm 0.5$ and $t_{3}=21^{\mathrm{d}} .0 \pm 0.5$. Clear glass plate and visual observations were omitted due to the high scatter. The decline for the first 60 days closely resembles a power law. In addition, this fit to the early data points gives an amazingly perfect extrapolation for the time after day 260 . The $V-I_{\mathrm{C}}$ color evolution shows that the temperature of the quasi-photosphere varied only between 4250 and $4900 \mathrm{~K}$ during the bump period, consistent with our spectra. At maximum, the temperature was $\approx 8100 \mathrm{~K}$ (Czart et al. 2006).

\section{Absolute magnitude, reddening and distance}

Nova distances can be derived from a comparison between their intrinsic and observed luminosity and therefore require that they are corrected for the effect of interstellar extinction. Van den Bergh \& Younger (1987) found that after correction for reddening, the intrinsic color of novae two magnitudes below maximum, i.e. at time $t_{2}$, is fairly constant $(B-V)_{0}=-0.02$, with a typical individual error (rms) of 0.12 . Although the coverage of $B$ values is sparse in our case, assuming a smooth behavior of $B$ like that in $V$, and $t_{2}=9 \pm 0^{\mathrm{d}} 5$, we have been able to derive $(B-V) @ t_{2}=0.58 \pm 0.03$ (see Fig. 1$)$ and thus $E(B-V)=$ $0.6 \pm 0.9$. Our value is in agreement with $E(B-V)=0.56$ from the interstellar Na I absorption lines (Siviero et al. 2006), 0.59 obtained from infrared observations (Russel et al. 2006),

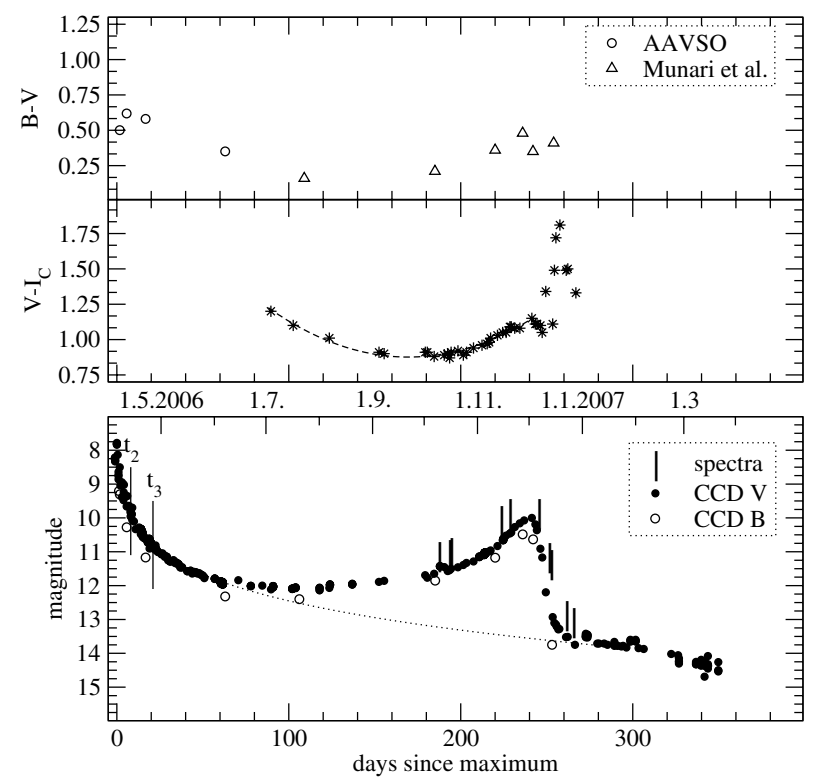

Fig. 1. The photometric data of V2362 Cyg from April 2006 to March 2007. The fit of a power law decline to data obtained the first 60 days is extrapolated to show the extension for the time after day 260 (dotted line). The dates of our spectral observations and of $t_{2}$ and $t_{3}$ used for the calculations in this paper are indicated.

0. 6 from fluorescently excited O I lines (Mazuk et al. 2006), and $0.65 \pm 0.05$ obtained using the progenitors colors and assuming a thick accretion disk spectrum (Steeghs et al. 2006). Thus, an $E(B-V)$ of $0.6 \pm 0.1\left(A_{\mathrm{V}}=1.9 \pm 0.3\right)$ is assumed throughout the paper.

Several methods for distance determinations of novae are suggested in the literature. The empirical relation between absolute magnitude at maximum and the rate of decline (DellaValle \& Livio 1995) leads to $M_{V} \approx-8.78 \pm 0$. 60 (using the conservative $3 \sigma$ boundary of the relation). With a corrected peak of $V_{0}(\mathrm{MAX})=5.93$, we derive a distance of $8.8_{-4}^{+9} \mathrm{kpc}$.

The standard candle method by Buscombe $\&$ de Vaucouleurs (1955) in the Warner (2003) calibration $M_{V}=-5.44$ fifteen days after visual maximum yields (the corrected magnitude was $\left.V_{15^{\mathrm{d}}}=8.55\right)$ to a distance of $6.4_{-2.5}^{+4.5} \mathrm{kpc}$. Finally, assuming a luminosity above Eddington at maximum as described in Bonifacio et al. (2000) we obtained $7.2_{-3}^{+5} \mathrm{kpc}$. We use a nonweighted mean of $D \approx 7.5_{-2.5}^{+3.0} \mathrm{kpc}$.

\section{The spectra}

Four types of spectra can be seen (Fig. 2). The spectra of October 2006 are dominated by the lines that have been visible throughout the whole normal decline starting in April 2006. The lines show the narrow structure, as reported by Siviero et al. (2006). Only $\mathrm{H}_{\alpha}$ starts to form a red shoulder of highvelocity material. The spectra taken on October 16 and 17, 2006 showed no differences and thus were averaged to improve $\mathrm{S} / \mathrm{N}$ for the further analysis. The spectra from November 2006 and December 7, 2006 are dominated by massive emissions of atoms at low excitation states. All show the wide red extension of the emission discussed in more detail for the hydrogen lines below. Most of the prominent lines show a fast blue P-Cygni absorption component at $\geq 2300 \mathrm{~km} \mathrm{~s}^{-1}$. The data taken on November 15 and 20, 2006 again have been combined into an average spectrum. By mid-December 2006 a transition spectrum emerged. Most of the nonforbidden lines have faded and the width of the 


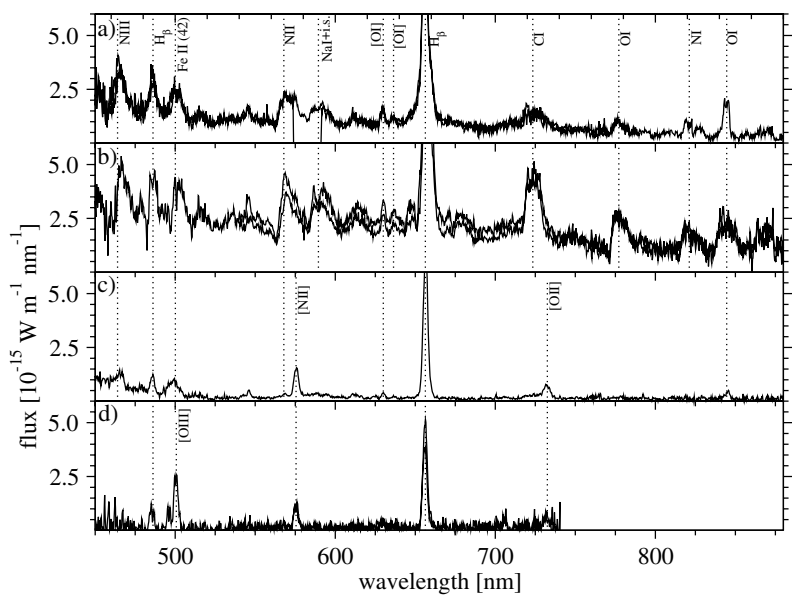

Fig. 2. The flux calibrated spectra: a) October 10 and 16/17, 2006 - start of the rebrightening; the spectra are nearly completely overlapping. b) November 15/20 and December 7, 2006 - at 2nd maximum; c) December 13/14, 2006 - transition phase; d) December 23 and 27, 2006 - nebular phase; only $\mathrm{H}_{\alpha}$ fades while the prominent forbidden lines are constant.

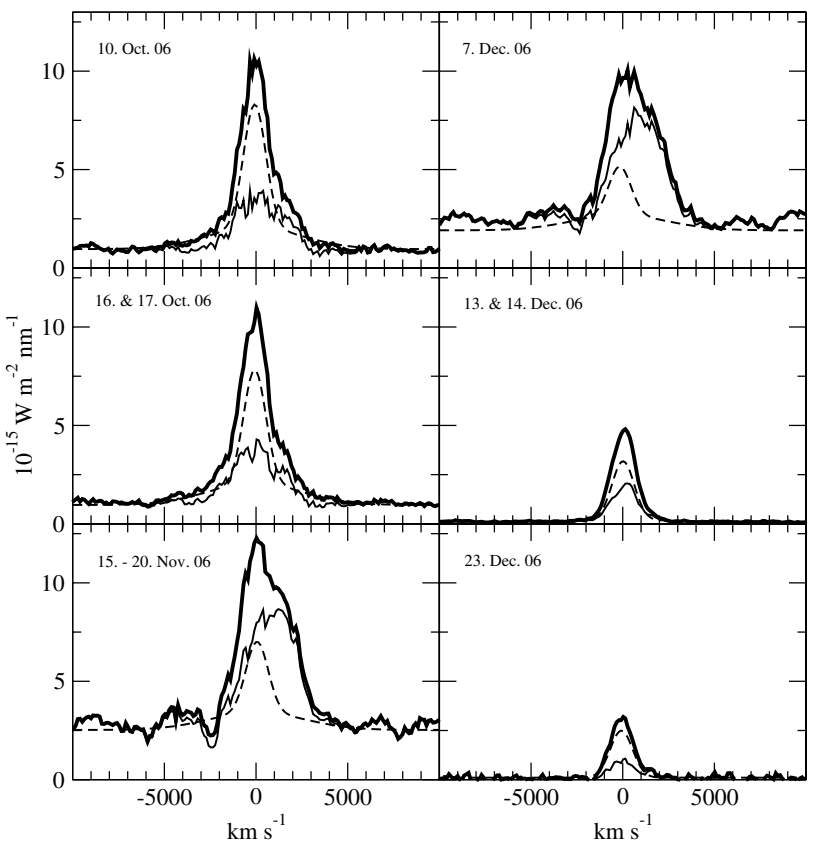

Fig. 3. The $\mathrm{H}_{\alpha}$ line illustrates the evolution of the different structures. The total emission (thick line), the fitted emission of the main outburst (dashed line) and the residual = component appearing only during the bump (thin line).

Balmer lines had decreased to their values before October 2006. Forbidden lines started to grow and finally dominated (except for $\mathrm{H}_{\alpha}$ ) the nebular spectra taken at the end of December. All spectra may be obtained electronically from the authors.

\section{The evolution of the $\mathrm{H}_{\alpha}$ line}

Due to the lower $\mathrm{S} / \mathrm{N}$ in the other lines, we focus exclusively on the remarkable line evolution using the strongest line, $\mathrm{H}_{\alpha}$ (Fig. 3). To gain insight into the nature of the bump event we fit the narrow emission using a simple optically-thin shell model arising from the main eruption. The bump event itself, far from being near the optically-thin regime, cannot be modeled without comprehensive radiative transfer calculations. Assuming an

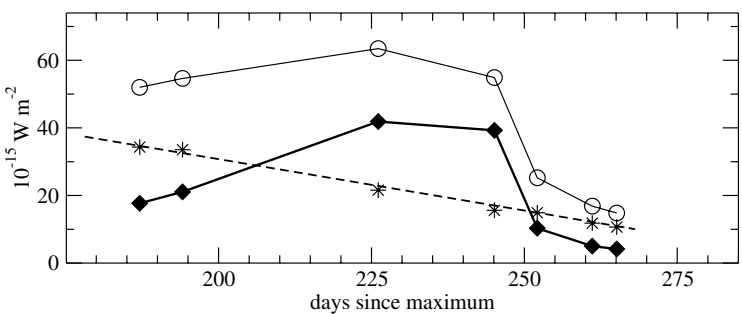

Fig. 4. The flux evolution of the total $\mathrm{H}_{\alpha}$ line flux (open circles), the fitted emission of the main outburst (asterisk), and the residual emission of the fast ejecta during the second maximum (filed diamonds).

instrument resolution folding of $0.6 \mathrm{~nm}$, the narrow emission profile perfectly fits that of two independent optically-thin expanding shells modeled after Lamers \& Cassinelli (1999), at the velocities reported by Siviero et al. (2006) and Munari et al. (2006b). The thickness of the shells are assumed to be negligible compared to their radii at this time. An integration along line of sight results in a normalized line profile $f(v ; \lambda)$ of a single shell is as function of the velocity $v$. The line profile for the detached shells expelled in April 2006 is

$g\left(v_{1}, v_{2}, a_{0}, r, c ; \lambda\right)=a_{0} \times\left(f\left(v_{1} ; \lambda\right)+r \times f\left(v_{1} ; \lambda\right)\right)+c$

where $v_{1}$ and $v_{2}$ are the expansion velocities of the shells, $c$ the level of the continuum. $a_{0}$ and $r$ are the fitting parameters for the total intensity and for the intensity ratio. This function was fitted to the data points $y_{i}$ between $\lambda_{\mathrm{MIN}}=\lambda_{0}-10.5 \mathrm{~nm}$ and $\lambda_{\text {MAX }}=\lambda_{0}+10.5 \mathrm{~nm}$. The velocities were fixed to those derived from the spectra of main eruption by Munari et al. (2006b; 1805 and $825 \mathrm{~km} \mathrm{~s}^{-1}$ respectively) to reduce the number of free parameters. With the boundary condition that there has to be no absorption redshifted from the maximum,

$\left[y_{i}-g\left(\lambda_{i}\right)\right] \geq c \quad \forall \lambda \geq \lambda_{0}=656.3 \mathrm{~nm}$

and the continuum level $c$ derived outside the wavelength range we used for the fit minimum of the logarithmic likelihood (Edwards 1992),

$I\left(a_{0}, r\right)=-\sum_{\lambda_{\mathrm{MIN}}}^{\lambda_{\mathrm{MAX}}} y_{i} \ln \left(\frac{g\left(a_{0}, r, c ; \lambda_{i}\right)}{\sum g\left(a_{0}, r, c ; \lambda_{i}\right)}\right)$

for the spectrum of October 10, 2006. The intensity ratio, $r$, was 0.78 on this date. As the main outburst ejecta were optically thin by this time, the shape and intensity ratio can be assumed to be constant for the other epochs. This assumption also avoids numerical instabilities from large numbers of free parameters. Note that the phenomenological model cannot provide absolute values for radii, densities, and physical conditions in these shells. The absolute minimum of the statistical likelihood is outside the physically-founded boundary; thus, a "goodness of fit" (e.g. by $I\left(a_{0}, r\right) \leq I_{\mathrm{MIN}}+0.5 \times n^{2}$, where $n$ gives the number of $\sigma_{\text {rms }}$ requested) cannot be derived.

The decline of the fitted component was remarkably smooth and homogenous, 1-2\% per day (Fig. 4), as is expected for completely detached shells. Fitting the remaining structure after subtracting the narrow emission model provides details on the bump emission. The newly formed shell had a velocity of about $2600 \mathrm{~km} \mathrm{~s}^{-1}$ and appeared early in October $2006\left(\approx 180^{\mathrm{d}}\right)$. Its emissivity peaked at the maximum of the brightening. 


\section{Discussion}

The derived ratio of $t_{2} / t_{3}$ and the decline rate of the first 60 days and 4.0 in brightness fit perfectly the normal decline described in Hachisu \& Kato $(2006,2007)$. In addition, this ratio also resembles the prediction from a linear fit through the novae with $t_{2}<20$ d 0 in Table 5.2 of Warner (2003). The correlation of the nova speed with the expansion velocities (McLaughlin 1960; Warner 2003) also resembles that of a normal system. Thus, it seems reasonable to use the samples in Hachisu \& Kato (2006, $2007)$ to estimate the white dwarf mass $M_{\mathrm{WD}}=1.2 \pm 0.1 M_{\odot}$ with $t_{3}$. Using the curves of Hachisu \& Kato (2001) leads to an even higher estimated mass. Although the samples mentioned above have a very large scatter of individual objects, they also give estimates for such mass for the accretor. On the other hand, even if the early decline looks very much like that of a normal object, one has to be dubious whether one is allowed to use such models for a nova with such a massive secondary maximum. Thus, the results here have to be estimates. For the proposed orbital period of 0.2070 (Goranskij et al. 2006) and the mass of the accreting white dwarf of $M_{\mathrm{WD}} \approx 1.2 M_{\odot}$ the secondary has to be a late type $M_{\mathrm{SE}} \leq 0.75 M_{\odot} \mathrm{K}$ to $\mathrm{M}$ star.

We used Cox (2000) to derive a temperature $T$ from stellar colors and the evolution of the luminosity $L$ from the lightcurve and the bolometric corrections. During the bump the temperature increased slightly from 4250 to $4900 \mathrm{~K}$ and decreased again at maximum to $4250 \mathrm{~K}$ (see $\left(V-I_{\mathrm{C}}\right)$ in Fig. 1). The temperature derived from the early spectra (Czart et al. 2006) was used as the temperature at maximum. Thereafter, the evolution of the radius of the "quasi" photosphere derived by $R \propto T^{-2} L^{0.5}$ was obtained. The shrinking of the photosphere stopped at day 60 and stayed constant at $15 \%$ of the radius of maximum until day 170 . In case of a homologous expansion this is only possible with a significant increase of density. The fast ejecta reached the photosphere around day 170 . With a speed of $2200 \mathrm{~km} \mathrm{~s}^{-1}$ - derived from our spectra and consistent with the value measured in November 2006 by Munari et al. (2006b) - and the estimated radius above, this bump material was accelerated about 45 to 60 days after maximum, assuming a negligibly small radius of the accelerating region. Subtracting the extrapolated power law decline and assuming that the bolometric correction derived by the color is constant at $\approx 0$. 12 to 0 . 2 , leads to an estimate of the radiative energy in the event. About $40 \%$ of the total radiated energy of the object during the whole outburst is contained in the bump. The high velocity of the fast ejecta during this epoch also significantly increased the mechanical energy. Hence, we presume that the bump event contained a significant fraction of the total outburst energy of the system.

The onset of massive dust formation, marked by the rapid decline, the fast rise of the color (see Fig. 1), and the IR excess (Rayner et al. 2006) around day 250 occurred most likely in the inner region only. Thus, the spectral components of the outer optically-thin regions continued with a smooth decline, while the fast component was obscured within a few days (see Fig. 4). The forbidden [OI] lines, which showed only the narrow component throughout the whole event, were generated in the outer region only. Although the obscured inner region, even seen later in the IR excess (Lynch et al. 2007), cannot directly heat the outer shell, an effective energy transport changed the excitation state quickly $-[\mathrm{OI}] \rightarrow[\mathrm{OII}] \rightarrow[\mathrm{OIII}]$ within 2 weeks.
The distance of $D \approx 7.5_{-2.5}^{+3.0} \mathrm{kpc}$ places V2362 Cyg at a galactocentric radius of about $11 \mathrm{kpc}$ and a distance to the plane of about $310 \mathrm{pc}$. As the direction marks the maximum of the Galactic warp above the plane (Momany et al. 2006), the real distance to the disk is about twice this value. This coincides with the spiral arm + II beyond the Perseus arm (Kimeswenger \& Weinberger 1989).

Further investigations of the ejecta of this unusual object and collection of all the data of various observers from the early decline until now is required to build the basis for a more sophisticated theoretical modeling. We would like to encourage modeling groups and would like to enliven a scientific discussion about the nature of the second outburst of V2362 Cyg and its older twin V1493 Aql.

Acknowledgements. We thank the referee Greg Schwarz for the extensive discussion improving the original manuscript. We acknowledge the variable star observations from the AAVSO International Database contributed by observers worldwide and used in this research.

\section{References}

Bonifacio, P., Selvelli, P. L., \& Caffau, E. 2000, A\&A, 356, L53

Buscombe, W, \& de Vaucouleurs, G. 1955, Obs., 75, 170

Cox, A. N. 2000, Allen's astrophysical quantities (New York: AIP Press; Springer)

Czart, K., Lewandowski, M., Maciejewski, G., et al. 2006, The Astronomer's Telegram, 792

Della Valle, M., \& Livio, M. 1995, ApJ, 452, 704

Edwards A. W. F. 1992, Likelihood (Baltimore: Johns Hopkins University Press) Goranskij, P. V., Metlova, V. N., \& Burenkov, N. A. 2006, The Astronomer's Telegram, 928

Hachisu, I., \& Kato, M. 2001, ApJ, 553, L161

Hachisu, I., \& Kato, M. 2006, ApJS, 167, 59

Hachisu, I., \& Kato, M. 2007, ApJ, 662, 552

Henden, A. A. 2007, Observations from the AAVSO International Database, private communication

Hernanz, M., Ferri, C., \& Sala, G. 2007, The Astronomer's Telegram, 1226 Kimeswenger, S., \& Weinberger, R. 1989, A\&A, 209, 51

Kimeswenger, S., Bacher, A., Emprechtinger, M., et al. 2003, A\&A, 409, 953

Kimeswenger, S., Dalnodar, S., Knapp, A., et al. 2006, The Astronomer's Telegram, 963

Lamers, H. J. G. L. M., \& Cassinelli, J. P. 1999, Introduction to Stellar Winds, Cambridge, (UK: Cambridge University Press)

Lynch, D. K., Russell, R. W., Kim, D., Sitko, M. L., \& Brafford, S. 2006, IAUC, 8785,2

Lynch, D. K., Rudy, R. J. Russell, R. W., et al. 2007, IAUC, 8849, 2

Mazuk, S., Rudy, R. J., Lynch, D. K., et al. 2006, IAUC, 8731, 2

McLaughlin, D. B. 1960, in Stellar Athmospheres, ed. J. L. Greenstein (Chicago University Press), 585

Momany, Y., Zaggia, S., Gilmore, G., et al. 2006, A\&A, 451, 515

Munari, U., Siviero, A., Valentini, M., et al. 2006a, CBET, 671, 1

Munari, U., Siviero, A., Navasardyan, H., et al. 2006b, CBET, 739, 1

Munari, U., Valentini, M., Siviero, A., et al. 2006c, CBET, 775

Nakano, S., Nishimura, H., Miles, R., \& Yamaoka, H. 2006, IAUC, 8697, 1

Ness, J.-U., Starrfield, S., Schwarz, G., et al. 2006, CBET, 696

Rayner, J., Rudy, R. J., Lynch, D. K., et al. 2006, IAUC, 8788, 1

Russell, R. W., Rudy, R. J., \& Lynch, D. K. 2006, IAUC, 8710, 2

Siviero, A., Munari, U., Valentini, M., \& Valisa, P. 2006, IAUC, 8702, 2

Steeghs, D., Greimel, R., Drew, J., et al. 2006, The Astronomer's Telegram, 795 van den Bergh, S., \& Younger, P. F. 1987, A\&AS, 70, 125

Warner, B. 2003, Cataclysmic Variable Stars, Cambridge (UK: Cambridge University Press)

Yamaoka, H. 2006, IAUC, 8702, 3

Yamaoka, H., Kinugasa, K., Naito, H., Ozaki, S., \& Fujii, M. 2006, IAUC, 8698,1 